\title{
Kolmogorov Numberings and Minimal Identification
}

\author{
Rusins Freivalds \\ Institute of Mathematics and Computer Science \\ University of Latvia \\ Raiņa bulvāris 29, LV-1459, Riga, Latvia \\ Email: rusins@mii.lu.lv \\ Sanjay Jain \\ Department of Information Systems and Computer Science \\ National University of Singapore \\ Lower Kent Ridge Rd \\ Singapore 119260 \\ Republic of Singapore \\ Email:sanjay@iscs.nus.sg
}

\begin{abstract}
Identification of programs for computable functions from their graphs by algorithmic devices is a well studied problem in learning theory. Freivalds and Chen consider identification of 'minimal' and 'nearly minimal' programs for functions from their graphs. To address certain problems in minimal identification for Gödel numberings, Freivalds later considered minimal identification in Kolmogorov Numberings. Kolmogorov numberings are in some sense optimal numberings and have some nice properties. We prove certain separation results for minimal identification in every Kolmogorov numbering. In addition we also compare minimal identification in Gödel numberings versus minimal identification in Kolmogorov numberings.
\end{abstract}

\section{Introduction}

Suppose $f$ is a total recursive function. For any natural number $n$, we let $f[n]$ denote $\{(x, f(x)) \mid x<n\}$, the finite initial segment of $f$ consisting of the first $n$ data points in the graph of $f$. Criteria of inference informally described below are formally defined in Section 3. In this paper we are only concerned about learnability of total recursive functions.

A function learning machine $\mathbf{M}$ is an algorithmic device which, on any input segment $f[n]$, returns either ? or a program. The output of $\mathbf{M}$ on $f[n]$ is denoted by $\mathbf{M}(f[n])$. If 
$\mathbf{M}(f[n])$ is a program, we think of that program as $\mathbf{M}$ 's conjecture, based on the data $f[n]$, about how to compute all of $f ; \mathbf{M}(f[n])=$ ? then represents the situation where $\mathbf{M}$ does not conjecture a program based on the data $f[n]$.

As is by now well known, there are various senses in which $\mathbf{M}$ can be thought of as successfully learning or inferring a program for $f$. Let $p_{n}=\mathbf{M}(f[n])$. The criterion of success known as Ex-identification [Gol67, BB75, CS83] requires that the sequence $p_{0}, p_{1}, p_{2}, \ldots$ contains a program $p$, which computes $f$, such that, for all but finitely many $i, p_{i}=p$. In this case one speaks of $p$ as being the final program output by $\mathbf{M}$ on $f$.

Based on the principle of Occam's Razor, Freivalds [Fre75] and Chen [Che81, Che82] studied the effect of requiring that the final hypothesis held by the learner in the above model be of (nearly) minimal size. Suppose $\psi$ is a numbering (programming system). In $\mathbf{M i n}_{\psi} \mathbf{E x}$-identification criterion one requires, in addition to Ex-identification of function (in the programming system $\psi$ ), that the final program be of minimal size (see formal definitions in Section 3). Minimal identification of classes of functions depends on the acceptable programming system (acceptable numbering) chosen to interpret programs output by learning machines. We direct the reader to [Fre75, Che81, Che82] for results dealing with minimal identification and its relationship with Ex-identification.

Freivalds [Fre75] showed that there are Gödel numberings in which no function class of infinite cardinality can be learned by minimal programs. This led Freivalds to consider minimal identification in Kolmogorov numberings. Kolmogorov numberings are numberings to which every other numbering is reducible by a linearly bounded function. Freivalds showed that for every Kolmogorov numbering $\psi$, there exists an infinite class of functions in $\mathbf{M i n}_{\psi} \mathbf{E x}$. In this paper we consider the relationship between identification criteria such as $\mathbf{M i n}_{\psi} \mathbf{E x}, \mathbf{M i n}_{\psi} \mathbf{F I N}$ and $\mathbf{M i n}_{\psi}$ CoLearn in Kolmogorov numberings $\psi$ (see formal definitions in Section 3). We show that these criteria are separated in every Kolmogorov numbering. We also show the existence of classes of functions which can be minimally identified in some Gödel numbering but cannot be minimally identified in any Kolmogorov numbering.

We now proceed formally.

\section{Notation}

Recursion-theoretic concepts not explained below are treated in [Rog67]. $N$ denotes the set of natural numbers, $\{0,1,2, \ldots\}$. The symbols $c, e, i, j, k, l, m, n, p, r, s, t, u, v$, $x, y$ and $z$, with or without decorations (decorations are subscripts, superscripts and the like), range over natural numbers unless otherwise specified. $\subseteq, \subset, \supseteq, \supset, \in$, denote subset, proper subset, superset, proper superset and membership relationship respectively. $\emptyset$ denotes the empty set. $A, C, L, S$, with or without decorations, range over subsets of $N$. $\bar{L}$ denotes the complement of $L$, i.e. $\bar{L}=N-L$. We denote the cardinality of a set $S$ by $\operatorname{card}(S) \cdot \max (), \min ()$ denote the maximum and minimum of a set, respectively. By convention $\max (\emptyset)=0$ and $\min (\emptyset)=\infty$. The quantifiers ' $\forall$ ' and ' $\exists$ ' mean 'for all but finitely many' and 'there exist infinitely many,' respectively. 
$\mathcal{R}$ denotes the set of all total recursive functions. $h, f, g, q$, with or without decorations, range over $\mathcal{R}$. $\mathcal{C}$ and $\mathcal{S}$, with or without decorations, range over subsets of $\mathcal{R}$. $\downarrow$ denotes defined. $\uparrow$ denotes undefined. $\xi$, with or without decorations, ranges over partial recursive functions.

A programming system (or computable numbering) is a (partial) computable function of two variables. We let $\psi, \beta, \eta$ range over computable numberings (programming systems). Suppose $\psi(\cdot, \cdot)$ is a computable numbering. We often refer to the (partial) function $\lambda x . \psi(i, x)$ as $\psi_{i}$. $\psi_{i}$ thus denotes the (partial) function computed by the $i$-th program in the numbering $\psi$. Note that, in general, a computable numbering may not contain all the partial recursive functions. We often drop the word 'computable' from 'computable numbering' in this paper, since we will be dealing with computable numberings only.

An acceptable numbering is a computable numbering to which every other computable numbering is reducible via a recursive function. Thus if $\psi$ is an acceptable numbering, then for all computable numberings $\eta$, there exists a recursive function $h$ such that $(\forall i)\left[\eta_{i}=\psi_{h(i)}\right]$. Acceptable numberings are also referred to as Gödel numberings. Kolmogorov numbering is an acceptable numbering to which every other computable numbering can be reduced via a linearly bounded function. Thus if $\psi$ is a Kolmogorov numbering, then for all computable numberings $\eta$, there exists a recursive function $h$ and a constant $c$ such that $(\forall i)\left[\eta_{i}=\psi_{h(i)} \wedge h(i) \leq \max (\{c * i, c\})\right]$.

For a function $f, \operatorname{MinProg}_{\psi}(f)$ denotes the minimal program for $f$, if any, in the $\psi$ programming system, i.e., $\operatorname{MinProg}_{\psi}(f)=\min \left(\left\{i \mid \psi_{i}=f\right\}\right)$. Let ZEROSTAR $=\{f \mid$ $(\forall x)[f(x)=0]\}$.

We let $\varphi$ denote an arbitrary fixed acceptable programming system. $\varphi_{i}$ thus denotes the partial recursive function computed by the $i^{\text {th }}$ program in the $\varphi$ acceptable programming system $\varphi$. We often refer to the $i^{\text {th }}$ program as program $i$. $\Phi$ denotes an arbitrary fixed Blum complexity measure [Blu67, HU79] for the $\varphi$-system.

$\lambda i, j .\langle i, j\rangle$ stands for an arbitrary computable one to one encoding of all pairs of natural numbers onto $N$ [Rog67]. We assume that the pairing function is such that $\langle i, j\rangle \geq \max (\{i, j\})$.

\section{Learning Paradigms}

For any partial function $\xi$ and any natural number $n$ such that, for each $x<n, \xi(x) \downarrow$, we let $\xi[n]$ denote the finite initial segment $\{(x, \xi(x)) \mid x<n\}$. Let INIT $=\{f[n] \mid f \in$ $\mathcal{R} \wedge n \in N\}$. We let $\sigma, \tau$ and $\gamma$, with or without decorations, range over INIT. We let $\Lambda$ denote the empty sequence. $|\sigma|$ denotes the length of $\sigma$. Thus for example $|f[n]|=n$.

Suppose $\xi$ is a partial function. Then zeroext $(\xi)$ denotes a function such that

$$
(\operatorname{zeroext}(\xi))(x)= \begin{cases}\xi(x), & \text { if } \xi(x) \downarrow ; \\ 0, & \text { otherwise. }\end{cases}
$$

Definition 1 [Gol67] A learning machine is an algorithmic device which computes a mapping from INIT into $N \cup\{?\}$ such that, if $\mathbf{M}(f[n]) \neq$ ?, then $\mathbf{M}(f[n+1]) \neq$ ?. 
In Definition 1 above, '?' denotes the situation when $\mathbf{M}$ outputs "no conjecture" on some $\sigma \in$ INIT. The restriction that $\mathbf{M}$ must continue to conjecture programs once it has done so is essentially without loss of generality since a machine which hasn't had enough time to think of a new conjecture can be thought of re-outputting its previous conjecture.

We let $\mathbf{M}$, with or without superscripts, range over learning machines. (We reserve $\mathbf{M}$ with subscripts for special type of enumeration of learning machines. See Proposition 7 below.)

Definition 2 Suppose $\mathbf{M}$ is a learning machine and $f$ is a computable function. $\mathbf{M}(f) \downarrow$ (read: $\mathbf{M}(f)$ converges) just in case $(\exists i)(\stackrel{\forall}{\forall} n)[\mathbf{M}(f[n])=i]$. If $\mathbf{M}(f) \downarrow$, then $\mathbf{M}(f)$ is defined as the unique $i$ such that $(\stackrel{\infty}{\forall} n)[\mathbf{M}(f[n])=i]$, otherwise we say that $\mathbf{M}(f)$ diverges (written: $\mathbf{M}(f) \uparrow)$.

We now formally define the criteria of inference considered in this paper.

\subsection{Explanatory Function Identification}

Definition 3 [Gol67, CS83] (a) A learning machine $\mathbf{M}$ is said to Ex-identify $f$ (written: $f \in \mathbf{E x}(\mathbf{M}))$ just in case $\left(\exists i \mid \varphi_{i}=f\right)[\mathbf{M}(f) \downarrow \wedge \mathbf{M}(f)=i]$.

(b) $\mathbf{E x}=\{\mathcal{C} \mid(\exists \mathbf{M})[\mathcal{C} \subseteq \mathbf{E x}(\mathbf{M})]\}$.

\subsection{Finite Function Inference}

Definition 4 [Gol67] (a) A learning machine $\mathbf{M}$ is said to FIN-identify $f$ (written: $f \in$ $\mathbf{F I N}(\mathbf{M}))$ just in case $\left(\exists n, p \mid \varphi_{p}=f\right)[(\forall m<n)[\mathbf{M}(f[m])=?] \wedge(\forall m \geq n)[\mathbf{M}(f[m])=$ $p]]$.

(b) $\mathbf{F I N}=\{\mathcal{C} \mid(\exists \mathbf{M})[\mathcal{C} \subseteq \mathbf{F I N}(\mathbf{M})]\}$

\subsection{Colearnability}

We say that $\mathbf{M}(f)$ co-converges to $p$, iff $[N-\{\mathbf{M}(f[m]) \mid m \in N\}=\{p\}]$. If there exists a $p$ such that $\mathbf{M}(f)$ co-converges to $p$, then we say that $\mathbf{M}$ co-converges on $f$ (to $p$ ). Otherwise we say that $\mathbf{M}$ co-diverges on $f$.

Definition 5 [FKS94] (a) A learning machine $\mathbf{M}$ is said to CoLearn $f$ (written: $f \in$ CoLearn $(\mathbf{M}))$ just in case $\left(\exists p \mid \varphi_{p}=f\right)[\mathbf{M}$ co-converges on $f$ to $p]$.

(b) CoLearn $=\{\mathcal{C} \mid(\exists \mathbf{M})[\mathcal{C} \subseteq \operatorname{CoLearn}(\mathbf{M})]\}$.

For the study of colearnability it is useful to define the following notation. Suppose $\sigma, \tau \in$ INIT. Then we define

$$
P_{\mathbf{M}}(\sigma, \tau)=\{\mathbf{M}(\gamma) \mid \mathbf{M}(\gamma) \neq ? \wedge \sigma \subseteq \gamma \subseteq \tau\}
$$


Intuitively $P_{\mathbf{M}}(\sigma, \tau)$ denotes the set of programs output by $\mathbf{M}$ on initial segments in $\{\gamma \mid \sigma \subseteq \gamma \subseteq \tau\}$

Similarly, we define

$$
P_{\mathbf{M}}(f[n], f)=\left\{\mathbf{M}\left(f\left[n^{\prime}\right]\right) \mid \mathbf{M}\left(f\left[n^{\prime}\right]\right) \neq ? \wedge n \leq n^{\prime}\right\}
$$

\subsection{Minimal Identification}

We next consider identification by minimal programs. Minimal identification usually depends on the numbering system chosen. Note that Ex, CoLearn and FIN are independent of acceptable programming system used. Thus without loss of generality, we have used the $\varphi$ acceptable programming system in the above definitions. However, minimal identification is acceptable programming system dependent, and thus we need to indicate the programming system in the definitions. For simplicity of presenting the proofs, we need to consider identification in non-acceptable programming systems also. Thus we consider a general definition of minimal identification, where the programming system used need not be acceptable.

Definition 6 [Fre75] Suppose $\psi$ is a numbering.

(a) $\mathbf{M}$ is said to $\mathbf{M i n}_{\psi} \mathbf{E x}$-identify $f$ (written $f \in \mathbf{M i n}_{\psi} \mathbf{E x}(\mathbf{M})$ ) iff $\mathbf{M}(f) \downarrow \wedge \mathbf{M}(f)=$ $\operatorname{MinProg}_{\psi}(f)$.

(b) $\operatorname{Min}_{\psi} \mathbf{E x}=\left\{\mathcal{C} \mid(\exists \mathbf{M})\left[\mathcal{C} \subseteq \mathbf{M i n}_{\psi} \mathbf{E x}(\mathbf{M})\right]\right\}$.

(c) $\mathbf{M}$ is said to $\mathbf{M i n}_{\psi} \mathbf{F I N}$-identify $f$ (written $\left.f \in \mathbf{M i n}_{\psi} \mathbf{F I N}(\mathbf{M})\right)$ iff $(\exists n)[(\forall m<$ $n)\left[\mathbf{M}(f[n])=\right.$ ?] $\left.\wedge(\forall m \geq n)\left[\mathbf{M}(f[n])=\operatorname{MinProg}_{\psi}(f)\right]\right]$.

(d) $\operatorname{Min}_{\psi} \mathbf{F I N}=\left\{\mathcal{C} \mid(\exists \mathbf{M})\left[\mathcal{C} \subseteq \operatorname{Min}_{\psi} \mathbf{F I N}(\mathbf{M})\right]\right\}$.

(e) $\mathbf{M}$ is said to $\mathbf{M i n}_{\psi}$ CoLearn $f$ (written $f \in \mathbf{M i n}_{\psi} \operatorname{CoLearn}(\mathbf{M})$ ) iff $\mathbf{M}(f)$ coconverges to $\operatorname{MinProg}_{\psi}(f)$.

(f) $\operatorname{Min}_{\psi}$ CoLearn $=\left\{\mathcal{C} \mid(\exists \mathbf{M})\left[\mathcal{C} \subseteq \operatorname{Min}_{\psi}\right.\right.$ CoLearn(M) $\left.]\right\}$.

The following proposition facilitates the proof of some of our results.

Proposition 7 There exists a recursively enumerable sequence $\mathbf{M}_{0}, \mathbf{M}_{1}, \ldots$ of learning machines such that, for all machines $\mathbf{M}$ and computable numberings $\psi$, there exists an $i$ such that, for $\mathbf{I} \in\left\{\mathbf{E x}, \mathbf{F I N}\right.$, CoLearn, $\mathbf{M i n}_{\psi} \mathbf{E x}, \mathbf{M i n}_{\psi}$ FIN, $\mathbf{M i n}_{\psi}$ CoLearn $\}$

$$
\mathbf{I}(\mathbf{M}) \subseteq \mathbf{I}\left(\mathbf{M}_{i}\right)
$$

For a proof of the above proposition see for example [OSW86]. We let $\mathbf{M}_{0}, \mathbf{M}_{1}, \ldots$ be one such enumeration.

FINITE denotes the collection of all finite classes of total recursive functions, i.e. FINITE $=\{\mathcal{C} \mid \mathcal{C} \subseteq \mathcal{R} \wedge \operatorname{card}(\mathcal{C})<\infty\}$. 


\section{Results}

\subsection{Relationship Between Minimal Inference Classes in Differ- ent Kolmogorov Numberings}

It is easy to see that for all acceptable numberings $\psi$, FINITE $\subseteq \operatorname{Min}_{\psi}$ FIN $\subseteq \operatorname{Min}_{\psi} \operatorname{CoLearn}_{\text {Co }}$ $\mathbf{M i n}_{\psi}$ Ex. In this section we show that FINITE, $\mathbf{M i n}_{\psi}$ FIN, Min ${ }_{\psi}$ CoLearn, $\mathbf{M i n}_{\psi} \mathbf{E x}$ differ for each Kolmogorov numbering $\psi$. Note that this is not the case for Gödel numberings since there exists a Gödel numbering $\eta$, such that no infinite class of functions is in $\operatorname{Min}_{\eta} \mathbf{E x}$.

Theorem 8 shows that $\operatorname{Min}_{\psi}$ FIN and FINITE differ for all Kolmogorov numberings; Theorem 11 shows that $\mathbf{M i n}_{\psi}$ CoLearn and $\mathbf{M i n}_{\psi}$ FIN differ for all Kolmogorov numberings; Theorem 14 shows that $\mathbf{M i n}_{\psi} \mathbf{E x}$ and $\mathbf{M i n}_{\psi}$ CoLearn differ for all Kolmogorov numberings.

Theorem $8(\forall$ Kolmogorov Numbering $\psi)\left[\operatorname{Min}_{\psi}\right.$ FIN - FINITE $\left.\neq \emptyset\right]$.

Proof. This is essentially the proof used by Freivalds [Fre91] to show that for every Kolmogorov numbering $\psi$, there exists an infinite class of functions in $\mathbf{M i n}_{\psi} \mathbf{E x}$. We give the proof for completeness. Suppose a Kolmogorov numbering $\psi$ is given. For $i>0$, let $h_{i}$ be defined as follows.

$$
h_{i}(x)= \begin{cases}i, & \text { if } x=0 \\ 0, & \text { otherwise }\end{cases}
$$

Let $c$ be such that,

$$
(\forall j>0)(\exists k \leq c * j)\left[\psi_{k}=h_{j}\right]
$$

Since $\psi$ is Kolmogorov numbering there exists such a $c$.

Let

$$
\mathcal{S}=\left\{h_{j} \mid j>0 \wedge \operatorname{card}\left(\left\{i \mid i \leq c * j \wedge \psi_{i}(0)=j\right\}\right) \leq 2 c\right\}
$$

It is easy to verify that $\mathcal{S}$ is infinite.

Let $C_{j}=\left\{k \mid k \leq c * j \wedge \psi_{k}(0)=j\right\}$. Let $p_{j}^{l}$ denote $l$-th element enumerated in some standard, 1-1, effective in $j$, enumeration of $C_{j}$.

Now for $1 \leq i \leq 2 c$, define $\mathbf{M}^{i}$ as follows. $\mathbf{M}^{i}$ on $h_{j}$ behaves as follows: If $p_{j}^{i}$ is defined, then $\mathbf{M}^{i}$ on $h_{j}$ outputs $p_{j}^{i}$ as its only program; otherwise, $\mathbf{M}^{i}$ does not output any program on $h_{j}$. Note that such $\mathbf{M}^{i}$ can easily be constructed.

Now it is easy to verify that,

$$
(\forall f \in \mathcal{S})(\exists l \mid 1 \leq l \leq 2 c)\left[f \in \mathbf{M i n}_{\psi} \mathbf{F I N}\left(\mathbf{M}^{i}\right)\right]
$$

Since $\mathcal{S}$ is infinite the theorem follows by Pigeon hole principle.

Definition 9 Suppose $\mathcal{C} \subseteq \mathcal{R}$ and $f \in \mathcal{C}$. Then $f$ is said to be an accumulation point for $\mathcal{C}$ iff $(\forall n)(\exists g \in \mathcal{C})[f \neq g \wedge(\forall x<n)[f(x)=g(x)]]$. 
The following Lemma is used in the proof of Theorem 11.

Lemma 10 Suppose $\mathcal{C} \subseteq \mathcal{R}$, and $f$ is an accumulation point for $\mathcal{C}$. Then $\mathcal{C} \notin \mathbf{F I N}$.

Proof. Let $\mathcal{C}$ and $f$ be given as in the hypothesis. Suppose by way of contradiction that M FIN-identifies $\mathcal{C}$. Then there exists an $n \in N$ such that $\mathbf{M}(f[n]) \neq$ ?. Let $g$ be such that $g \neq f$ and $(\forall x \leq n)[f(x)=g(x)]$. Then $\mathbf{M}$ fails to FIN-identify at least one of $g$ and $f$. A contradiction. Thus no such $\mathbf{M}$ can exist.

\section{Theorem $11(\forall$ Kolmogorov Numbering $\psi)\left[\operatorname{Min}_{\psi}\right.$ CoLearn - FIN $\left.\neq \emptyset\right]$.}

Proof. Suppose a Kolmogorov numbering $\psi$ is given. Let $h_{0}$ be everywhere 0 function. For $i>0$, define $h_{i}$ as follows.

$$
h_{i}(x)= \begin{cases}1, & \text { if } x=i \\ 0, & \text { otherwise }\end{cases}
$$

We will construct a class of functions $\mathcal{C} \in \operatorname{Min}_{\psi}$ CoLearn, such that $h_{0} \in \mathcal{C}$ and for infinitely many $k, h_{k} \in \mathcal{C}$. Note that $h_{0}$ would thus be an accumulation point for $\mathcal{C}$. This, using Lemma 10, would imply the Theorem.

Let $z=\operatorname{MinProg}_{\psi}\left(h_{0}\right)$.

Let $c$ be such that $(\forall k>0)\left[\operatorname{MinProg}_{\psi}\left(h_{k}\right) \leq c * k\right]$ (note that since $\psi$ is a Kolmogorov numbering, there exists such a $c)$. For $k>0$, let $C_{k}=\left\{j \mid j \leq c * k \wedge h_{k}[k+1] \subseteq \psi_{j}\right\}$. Let $p_{k}^{l}$ denote the $l$-th program enumerated, if any, in some standard, $1-1$, effective in $k$, enumeration of $C_{k}$.

Let $c^{\prime}$ be a constant $>2$. For $l \in N^{+}$, consider machine $\mathbf{M}^{l}$, such that the following two properties are satisfied.

(1) $P_{\mathbf{M}^{l}}\left(\Lambda, h_{0}[y]\right)=\left\{i \mid i \leq y / c^{\prime}\right\}-\{z\}$.

(Note that this implies that $h_{0} \in \operatorname{Min}_{\psi} \operatorname{CoLearn}\left(\mathbf{M}^{l}\right)$.)

$$
P_{\mathbf{M}^{l}}\left(h_{k}[k+1], h_{k}\right)= \begin{cases}N-\left\{p_{k}^{l}\right\}, & \text { if } l \leq \operatorname{card}\left(C_{k}\right) \\ \{z\}, & \text { if } l>\operatorname{card}\left(C_{k}\right) .\end{cases}
$$

Note that one can easily construct such $\mathbf{M}^{l}$.

Let

$$
S=\left\{k \mid k>0 \wedge \operatorname{card}\left(C_{k}\right) \leq 2 c\right\}-\left\{k \mid k>0 \wedge \operatorname{MinProg}_{\psi}\left(h_{k}\right) \leq k / c^{\prime}\right\}
$$

It is easy to verify that $S$ is of infinite cardinality (since $c^{\prime}>2$ ). Furthermore, $(\forall k \in S)(\exists l \mid 1 \leq l \leq 2 c)\left[h_{k} \in \operatorname{Min}_{\psi} \operatorname{CoLearn}\left(\mathbf{M}^{l}\right)\right]$. It follows, using Pigeon hole principle, that there exists an $l, 1 \leq l \leq 2 c$, such that $\operatorname{Min}_{\psi} \operatorname{CoLearn}\left(\mathbf{M}^{l}\right)$ contains an infinite subset of $\left\{h_{k} \mid k>0\right\}$. Since $h_{0} \in \operatorname{Min}_{\psi} \operatorname{CoLearn}\left(\mathbf{M}^{l}\right)$, it follows that there exists a $\mathcal{C} \in \operatorname{Min}_{\psi}$ CoLearn, such that $h_{0} \in \mathcal{C}$, and for infinitely many $k, h_{k} \in \mathcal{C}$, as claimed. 
Note that $\mathbf{F I N}-\operatorname{Min}_{\psi}$ CoLearn $\neq \emptyset$, for all acceptable numbering $\psi .(\mathcal{C}=\{f \mid$ $(\forall x>0)[f(x)=0]\}$, witnesses the separation.)

As corollaries we have

Corollary $12(\forall$ Kolmogorov Numbering $\psi)(\exists \mathcal{C})\left[\mathcal{C} \in \operatorname{Min}_{\psi}\right.$ CoLearn $\wedge(\forall$ Gödel Numbering $\left.\eta)\left[\mathcal{C} \notin \operatorname{Min}_{\eta} \mathbf{F I N}\right]\right]$.

\section{Corollary $13(\forall$ Kolmogorov Numbering $\psi)\left[\operatorname{Min}_{\psi} \operatorname{CoLearn}-\operatorname{Min}_{\psi} \mathbf{F I N} \neq \emptyset\right]$.}

Theorem $14(\forall \quad$ Kolmogorov Numbering $\psi)(\exists \mathcal{C})\left[\mathcal{C} \in \mathbf{M i n}_{\psi} \mathbf{E x} \wedge(\forall \quad\right.$ Kolmogorov Numbering $\left.\psi^{\prime}\right)\left[\mathcal{C} \notin \operatorname{Min}_{\psi^{\prime}}\right.$ CoLearn $]$.

PROOF. Let $\beta^{n}$ denote the $n$-th Kolmogorov numbering (in some ordering of Kolmogorov numberings; note that we do not need the ordering to be effective - in fact any such ordering will not be effective).

We first define a computable numbering $\eta$ (an appropriate subset of functions computed by the programs in the numbering $\eta$ will serve as our diagonalizing class).

Intuitively, we consider the programs of $\eta$ to be divided in different groups $G_{i}^{j}=\{p \mid$ $\left.l_{i}^{j} \leq p \leq u_{i}^{j}\right\}$, where $j \leq i$, and $l_{i}^{j}, u_{i}^{j}$ are defined below. For each $i$, for some $s_{i} \leq i$, $G_{i}^{s_{i}}$ will provide us with a (large) set of functions $\mathcal{S}_{i}$, such that, for each $k, n \in N$, for large enough $i$, at most one of the functions in $\mathcal{S}_{i}$ belongs to $\operatorname{Min}_{\beta^{n}} \operatorname{CoLearn}\left(\mathbf{M}_{k}\right)$. This will allow us construct our diagonalizing class $\mathcal{C}$ using techniques similar to that used in earlier theorems of this paper. We now proceed formally.

Let $l_{0}^{0}=0, u_{0}^{0}=0$.

For $i>0$,

$$
\begin{aligned}
& \text { let } l_{i}^{0}=u_{i-1}^{i-1}+1 \\
& \text { for } 1 \leq j \leq i, \text { let } l_{i}^{j}=u_{i}^{j-1}+1 .
\end{aligned}
$$

For $i>0, j \leq i$, let $u_{i}^{j}=\left(l_{i}^{j}+i^{2}\right) * 2$.

For $l_{i}^{0} \leq r \leq u_{i}^{i}$, we define $\eta_{r}$ according to the following staging construction (note that for each $i$, a different such staging construction, effective in $i$, is executed).

Let $\sigma_{i}^{0}=\{(0, i)\}$. Go to stage 0 .

Stage $s$

For $l_{i}^{s} \leq r \leq u_{i}^{s}$, let

$$
\eta_{r}(x)= \begin{cases}\sigma_{i}^{s}(x), & \text { if } x<\left|\sigma_{i}^{s}\right| \\ r, & \text { if } x=\left|\sigma_{i}^{s}\right| \\ 0, & \text { otherwise }\end{cases}
$$

Search for a $r, n$ such that

$$
\begin{aligned}
& l_{i}^{s} \leq r \leq u_{i}^{s}, \\
& n>\left|\sigma_{i}^{s}\right|, \text { and } \\
& \operatorname{card}\left(\left\{k \mid k<i \wedge \operatorname{card}\left(\left\{x \mid x \leq i * u_{i}^{i}\right\}-P_{\mathbf{M}_{k}}\left(\Lambda, \eta_{r}[n]\right)\right) \leq 1\right\}\right) \geq s+1 .
\end{aligned}
$$


(Note that the success of above search means that at least $s+1$ of the machines in $\mathbf{M}_{0}, \mathbf{M}_{1}, \ldots, \mathbf{M}_{i-1}$, output all but possibly one of the programs $\leq i * u_{i}^{i}$ on initial segments of $\left.\eta_{r}[n].\right)$

If and when such $r, n$ are found, let $\sigma_{i}^{s+1}=\eta_{r}[n]$, and go to stage $s+1$.

End Stage $s$

Note that for any $i$, the last stage executed is $\leq i$ (since, $\operatorname{card}(\{k \mid k<i \wedge \operatorname{card}(\{x \mid$ $\left.\left.\left.\left.x \leq i * u_{i}^{i}\right\}-P_{\mathbf{M}_{k}}\left(\Lambda, \eta_{r}[n]\right)\right) \leq 1\right\}\right)$ is bounded by $\left.i\right)$.

For $i \in N$, let $s_{i}$ denote the last stage executed in the above construction corresponding to $i$.

Claim 15 (a) Suppose $i \in N, j \leq s_{i}$, and $l_{i}^{j} \leq r \leq u_{i}^{j}$. Then $\eta_{r} \in$ ZEROSTAR and $\eta_{r}\left(\max \left(\left\{x \mid \eta_{r}(x) \neq 0\right\}\right)\right)=r$.

(b) Suppose $i \in N, s_{i}<j \leq i$, and $l_{i}^{j} \leq r \leq u_{i}^{j}$. Then $\eta_{r}$ is everywhere undefined.

(c) If $r \neq r^{\prime}$, then $\eta_{r}=\eta_{r^{\prime}}$ implies $\eta_{r}$ is everywhere undefined.

Proof. Parts (a) and (b) follow from definition of $\eta_{r}$ and $s_{i}$. Part (c) follows from parts (a) and (b).

Let $\mathcal{S}_{i}=\left\{\eta_{r} \mid l_{i}^{s_{i}} \leq r \leq u_{i}^{s_{i}}\right\}$.

Let $c_{n}$ be such that $(\forall r>0)\left[\operatorname{MinProg}_{\beta^{n}}\left(\eta_{r}\right) \leq c_{n} * r\right]$ (such $c_{n}$ exist since $\beta^{n}$ is a Kolmogorov numbering).

Let $E_{k, n}^{i}=\left\{f \mid f \in \mathcal{S}_{i} \wedge c_{n} \leq i \wedge f \in \operatorname{Min}_{\beta^{n}} \operatorname{CoLearn}\left(\mathbf{M}_{k}\right)\right\}$.

Claim 16 Let $k, n \in N . \operatorname{Min}_{\beta^{n}} \operatorname{CoLearn}\left(\mathbf{M}_{k}\right)$ contains at most finitely many functions in $\left[\bigcup_{i} \mathcal{S}_{i}\right]-\left[\bigcup_{\{i, k, n \mid k, n<i\}} E_{k, n}^{i}\right]$.

Proof. Follows from the definition of $E_{k, n}^{i}$.

Claim 17 Suppose $k, n<i$. Then $\operatorname{card}\left(E_{k, n}^{i}\right) \leq 1$.

Proof. Suppose $k, n<i$, and $c_{n} \leq i$. Now for all $f \in \mathcal{S}_{i}, \operatorname{MinProg}_{\beta^{n}}(f) \leq i * u_{i}^{i}($ since $\left.\operatorname{MinProg}_{\eta}(f) \leq u_{i}^{i}\right)$. Also for all $f \in \mathcal{S}_{i}, \sigma_{i}^{s_{i}} \subseteq f$.

Let $X=\left\{k^{\prime} \mid k^{\prime}<i \wedge \operatorname{card}\left(\left\{x \mid x \leq i * u_{i}^{i}\right\}-P_{\mathbf{M}_{k^{\prime}}}\left(\Lambda, \sigma_{i}^{s_{i}}\right)\right) \leq 1\right\}$.

Now we consider two cases:

Case 1: $k \notin X$.

Note that in this case, we have that $\mathbf{M}_{k}(f)$ co-diverges on all $f \in \mathcal{S}_{i}$ (otherwise the search in stage $s_{i}$ of the construction above would have succeeded). Thus $\operatorname{card}\left(E_{k, n}^{i}\right)=0$.

Case 2: $k \in X$.

In this case since,

$$
\operatorname{card}\left(\left\{x \mid x \leq i * u_{i}^{i}\right\}-P_{\mathbf{M}_{k}}\left(\Lambda, \sigma_{i}^{s_{i}}\right)\right) \leq 1
$$

we have that $\mathbf{M}_{k}$ can $\mathbf{M i n}_{\beta^{n}}$ CoLearn at most one $f \in \mathcal{S}_{i}$. Thus card $\left(E_{k, n}^{i}\right) \leq$ 1. 
Now suppose $\psi$ is a Kolmogorov numbering. Let $c$ be such that $(\forall l>0)\left[\operatorname{MinProg}_{\psi}\left(\eta_{l}\right) \leq\right.$

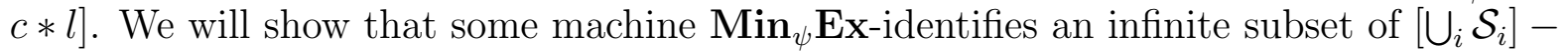
$\left[\bigcup_{\{i, k, n \mid k, n<i\}} E_{k, n}^{i}\right]$. This would prove the theorem (using Claim 16).

Let $\mathcal{S}=\left[\bigcup_{i} \mathcal{S}_{i}\right]-\left[\left[\bigcup_{\{i, k, n \mid k, n<i\}} E_{k, n}^{i}\right] \cup\left\{\eta_{r} \mid(\exists i)\left[l_{i}^{s_{i}} \leq r \leq u_{i}^{s_{i}}\right] \wedge \operatorname{card}(\{p \mid p \leq\right.\right.$ $\left.\left.\left.\left.c * r \wedge \eta_{r}\left[\left|\sigma_{i}^{s_{i}}\right|+1\right] \subseteq \psi_{p}\right\}\right)>3 c\right\}\right]$. It is easy to verify that $\mathcal{S}$ is infinite (for $u_{i}^{i}>c$, at least $2 u_{i}^{s_{i}} / 3-l_{i}^{s_{i}}-i^{2}$ of the functions in $\mathcal{S}_{i}$ belong to $\left.\mathcal{S}\right)$.

For $l_{i}^{s_{i}} \leq r \leq u_{i}^{s_{i}}$, let $C_{r}=\left\{r^{\prime} \mid r^{\prime} \leq c * r \wedge \eta_{r}\left[\left|\sigma_{i}^{s_{i}}\right|+1\right] \subseteq \psi_{r^{\prime}}\right\}$. Let $p_{r}^{l}$ denote the $l$-th program, if any, enumerated in some standard, 1-1, effective in $r$, enumeration of $C_{r}$.

For $1 \leq l \leq 3 c$, let $\mathbf{M}^{l}$ be such that,

$$
(\forall i)\left(\forall r \mid l_{i}^{s_{i}} \leq r \leq u_{i}^{s_{i}}\right)\left[\mathbf{M}^{l}\left(\eta_{r}\right)=p_{r}^{l}\right]
$$

Note that such $\mathbf{M}^{l}$ can easily be constructed. It is easy to verify that, for each $\eta_{r} \in \mathcal{S}$, there exists an $l, 1 \leq l \leq 3 c$ such that $\eta_{r} \in \mathbf{M i n}_{\psi} \mathbf{E x}\left(\mathbf{M}^{l}\right)$. Thus, by pigeon hole principle, there exists an infinite subset of $\mathcal{S}$ in $\mathbf{M i n}_{\psi} \mathbf{E x}$. Since $\mathcal{S} \subseteq\left[\bigcup_{i} \mathcal{S}_{i}\right]-\left[\bigcup_{\{i, k, n \mid k, n<i\}} E_{k, n}^{i}\right]$,

we have that, an infinite subset of $\left[\bigcup_{i} \mathcal{S}_{i}\right]-\left[\bigcup_{\{i, k, n \mid k, n<i\}} E_{k, n}^{i}\right]$ belongs to $\mathbf{M i n}_{\psi} \mathbf{E x}$. The theorem follows using Claim 16.

A modification of the above proof can be used to show that

Theorem $18(\forall$ Kolmogorov Numbering $\psi)(\exists \mathcal{C})\left[\mathcal{C} \in \mathbf{M i n}_{\psi} \mathbf{E x} \wedge(\forall\right.$ Gödel Numbering $\left.\psi^{\prime}\right)\left[\mathcal{C} \notin \operatorname{Min}_{\psi^{\prime}}\right.$ CoLearn $\left.]\right]$.

We omit the details. As a corollary to Theorem 14 we have,

Corollary $19(\forall$ Kolmogorov Numbering $\psi)\left[\operatorname{Min}_{\psi} \mathbf{E x}-\mathbf{M i n}_{\psi}\right.$ CoLearn $\left.\neq \emptyset\right]$.

As a Corollary Theorem 8 and Corollaries 13 and 19 we have

Corollary $20(\forall$ Kolmogorov Numbering $\psi)\left[\right.$ FINITE $\subset$ Min $_{\psi}$ FIN $\subset$ Min $_{\psi}$ CoLearn $\subset$ $\left.\operatorname{Min}_{\psi} \mathbf{E x}\right]$.

\subsection{Recursively Enumerable Classes and Minimal Identification in Kolmogorov/Gödel Numberings}

The next three theorems consider the question about whether recursively enumerable classes can be minimally identified in Gödel or Kolmogorov numberings.

Theorem 21 ( $\exists$ infinite r.e. $\mathcal{C})(\exists$ Kolmogorov Numbering $\psi)\left[\mathcal{C} \in \mathbf{M i n}_{\psi} \mathbf{E x}\right]$.

PROOF. It was shown in [Fre91] that there exists a Kolmogorov numbering $\psi$ such that $\{f \mid(\forall x)[f(x)=f(0)]\} \in \mathbf{M i n}_{\psi} \mathbf{E x}$. In fact it can be shown that for all $\mathcal{C} \in \mathbf{F I N}$, there exists a Kolmogorov numbering $\psi$, such that $\mathcal{C} \in \mathbf{M i n}_{\psi} \mathbf{E x}$. 
Theorem $22(\forall$ infinite r.e. $\mathcal{C})(\forall$ Gödel Numbering $\psi)\left[\mathcal{C} \notin \operatorname{Min}_{\psi}\right.$ CoLearn $]$.

Proof. Suppose by way of contradiction that $\mathbf{M}$, an r.e. infinite class $\mathcal{C}$, and a Gödel Numbering $\psi$, are such that $\mathcal{C} \subseteq \mathbf{M i n}_{\psi} \operatorname{CoLearn}(\mathbf{M})$.

It follows that, for all $c$, there exists an $f \in \mathcal{C}$ such that $P_{\mathbf{M}}(\Lambda, f) \supseteq\{x \mid x \leq c\}$. Note that, since $\mathcal{C}$ is r.e., one can search, effectively in $c$, for such a function $f$.

Thus, by implicit use of Kleene's recursion theorem [Rog67], there exists an $e$, such that $\psi_{e} \in \mathcal{C}$, and $P_{\mathbf{M}}\left(\Lambda, \psi_{e}\right) \supseteq\{x \mid x \leq e\}$. But then $\psi_{e} \notin \operatorname{Min}_{\psi} \operatorname{CoLearn}(\mathbf{M})$. A contradiction. Thus $(\forall$ infinite r.e. $\mathcal{C})(\forall$ Gödel Numbering $\psi)\left[\mathcal{C} \notin \operatorname{Min}_{\psi}\right.$ CoLearn $]$.

Theorem 23 ( $\exists$ Kolmogorov Numbering $\psi)(\exists$ infinite co-r.e. $L)\left[\left\{\psi_{i} \mid i \in L\right\} \in\right.$ $\left.\operatorname{Min}_{\psi} \mathbf{F I N}\right]$.

Proof. Let $f_{k}$ be defined as follows.

$$
f_{k}(x)= \begin{cases}k, & \text { if } x=0 \\ 0, & \text { otherwise }\end{cases}
$$

Without loss of generality suppose that $\varphi$ is a Kolmogorov numbering.

Let $\psi$ be defined as follows.

$$
\psi_{j}= \begin{cases}\varphi_{i}, & \text { if } j=3 i \\ f_{j}, & \text { if } j \text { is not divisible by } 3 .\end{cases}
$$

Let

$$
\bar{L}=\{3 i \mid i \in N\} \cup\left\{j \mid j \text { is not divisible by } 3 \wedge(\exists i<j / 3)\left[\varphi_{i}(0)=j\right]\right\}
$$

Note that $\bar{L}$ is r.e. and coinfinite. Consider $\mathbf{M}$, which on $f_{k}$, outputs $k$ as its only program. It is easy to verify that $\mathcal{C}=\left\{\psi_{j} \mid j \in L\right\} \subseteq \mathbf{M i n}_{\psi} \mathbf{F I N}(\mathbf{M})$.

\subsection{Minimal Identification in Gödel Numberings vs. Kolmogorov Numberings}

In this section we compare the effects of considering Gödel numberings versus Kolmogorov numbering on minimal identification. Specifically, we show that, for each of the three identification types, FIN, CoLearn, Ex, discussed in this paper, there exists a class of functions, $\mathcal{C}$, which can be identified using minimal programs in some Gödel numbering but cannot be identified using minimal programs in any Kolmogorov numbering.

Theorem $24(\exists$ Gödel Numbering $\psi)(\exists \mathcal{C})\left[\mathcal{C} \in \operatorname{Min}_{\psi} \mathbf{F I N} \wedge(\forall\right.$ Kolmogorov Numbering $\left.\psi^{\prime}\right)\left[\mathcal{C} \notin \operatorname{Min}_{\psi^{\prime}}\right.$ CoLearn] $]$. 
Proof. Let $h_{i}$ be a function defined as follows.

$$
h_{i}(x)= \begin{cases}i, & \text { if } x=0 \\ 0, & \text { otherwise }\end{cases}
$$

Let $\psi_{i}$ be defined as follows:

$$
\psi_{i}= \begin{cases}\varphi_{l}, & \text { if } i=l^{4} \\ h_{i}, & \text { if for all } l, i \neq l^{4} .\end{cases}
$$

Clearly, $\psi$ is a Gödel numbering. Consider $\mathbf{M}^{\prime}$, which on $h_{i}$, outputs $i$ as its only program. Let $\mathcal{C}=\left\{h_{i} \mid \operatorname{MinProg}_{\psi}\left(h_{i}\right)=i\right\}$. Clearly, $\mathcal{C} \subseteq \operatorname{Min}_{\psi} \mathbf{F I N}\left(\mathbf{M}^{\prime}\right)$.

Claim 25 At most $r+1$ of the functions in $\left\{h_{i} \mid r^{4}<i<(r+1)^{4}\right\}$ are not in $\mathcal{C}$.

Proof. Note that $\operatorname{card}\left(\left\{i \mid i<(r+1)^{4} \wedge \psi_{i} \neq h_{i}\right\}\right) \leq r+1$. Thus since $h_{i}$ 's are distinct, $\operatorname{card}\left(\left\{i \mid r^{4}<i<(r+1)^{4} \wedge \operatorname{MinProg}_{\psi}\left(h_{i}\right) \neq i\right\}\right) \leq r+1$. Claim follows.

Suppose $\mathbf{M}$ and a Kolmogorov numbering $\psi^{\prime}$ is given. We will show that $\mathcal{C} \nsubseteq$ $\operatorname{Min}_{\psi^{\prime}} \operatorname{CoLearn}(\mathbf{M})$. To show this we, construct a recursive function $q$ (using operator recursion theorem [Cas74]) such that, for some $i, \varphi_{q(i)}$ has a small enough program in $\psi^{\prime}$, but $\mathbf{M}$ on $\varphi_{q(i)}$ does not co-converge to a small enough program.

By Operator recursion theorem [Cas74], there exists a recursive function $q$, such that (partial) functions $\varphi_{q(\cdot)}$ may be defined as follows.

Let $l_{0}=0 . l_{s}$ is used to denote the least $l$ such that $\varphi_{q(l)}$ has not been defined on any input before stage $s$. Go to stage 0 .

Begin stage $s$

1. Search for an $r>l_{s}, S \subseteq\left\{x \mid r^{4}<x<(r+1)^{4}\right\}$, such that $\operatorname{card}(S)=r+2$ and, for all $i \in S,\left\{x \mid x \leq\left(l_{s}+r+2\right)^{2}\right\} \subseteq P_{\mathbf{M}}\left(\Lambda, h_{i}\right)$.

2. Let $S$ be as found in step 1. Let $\varphi_{q\left(l_{s}+x\right)}, x \leq r+1$, be the $r+2$ functions in $\left\{h_{i} \mid i \in S\right\}$.

3. Let $l_{s+1}=l_{s}+r+2$.

End stage $s$

Now we consider the following cases.

Case 1: Stage $s$ starts but does not halt.

Non success of the search at step 1 , implies that, for any $r>l_{s}, \mathbf{M}$ can colearn (in numbering $\psi$ ) at most $\left(l_{s}+r+2\right)^{2}+r+1 \leq(2 r+2)^{2}+r+1$ of the functions in $\left\{h_{i} \mid r^{4}<i<(r+1)^{4}\right\}$. Now, for each $r$, by Claim 25, at least $(r+1)^{4}-r^{4}-1-(r+1)$ of the functions in $\left\{h_{i} \mid r^{4}<i<(r+1)^{4}\right\}$, are in $\mathcal{C}$.

Thus, since $(r+1)^{4}-r^{4}-1-(r+1)>(2 r+2)^{2}+r+1$, for large enough $r$, non-success of step 1 in stage $s$ implies $\mathcal{C} \nsubseteq \mathbf{M i n}_{\psi^{\prime}} \operatorname{CoLearn}(\mathbf{M})$.

Case 2: All stages halt. 
In this case note that, by Claim 25, for each stage, at least one of the $r+2$ functions found in step 1 is in $\mathcal{C}$. It follows that $(\stackrel{\infty}{\exists} i)\left[\varphi_{q(i)} \in \mathcal{C} \wedge \mathbf{M}\right.$ on $\varphi_{q(i)}$ does not co-converge to a program $\left.\leq i^{2}\right]$. But since, for some constant $c$, for all $i, \operatorname{MinProg}_{\psi^{\prime}}\left(\varphi_{q(i)}\right) \leq c * i$, we have

$$
(\stackrel{\infty}{\exists} i)\left[\varphi_{q(i)} \in\left(\mathcal{C}-\operatorname{Min}_{\psi^{\prime}} \operatorname{CoLearn}(\mathbf{M})\right)\right]
$$

Thus $\mathcal{C} \nsubseteq \operatorname{Min}_{\psi^{\prime}} \operatorname{CoLearn}(\mathbf{M})$.

As corollaries we have,

Corollary $26(\exists \mathcal{C})(\exists$ Gödel Numbering $\psi)\left[\mathcal{C} \in \operatorname{Min}_{\psi} \mathbf{F I N} \wedge(\forall\right.$ Kolmogorov Numbering $\left.\eta)\left[\mathcal{C} \notin \operatorname{Min}_{\eta} \mathbf{F I N}\right]\right]$.

Corollary $27(\exists \mathcal{C})(\exists \quad$ Gödel Numbering $\psi)\left[\mathcal{C} \in \operatorname{Min}_{\psi}\right.$ CoLearn $\wedge(\forall$ Kolmogorov Numbering $\eta)\left[\mathcal{C} \notin \operatorname{Min}_{\eta}\right.$ CoLearn $\left.]\right]$.

Note that we cannot strengthen the above theorem to show, ( $\exists$ Gödel Numbering $\psi)(\exists \mathcal{C})\left[\mathcal{C} \in \operatorname{Min}_{\psi} \mathbf{F I N} \wedge\left(\forall\right.\right.$ Kolmogorov Numbering $\left.\left.\psi^{\prime}\right)\left[\mathcal{C} \notin \mathbf{M i n}_{\psi^{\prime}} \mathbf{E x}\right]\right]$. This is so because, $(\forall \mathcal{C} \in \mathbf{F I N})\left[\left(\exists\right.\right.$ Kolmogorov Numbering $\left.\left.\psi^{\prime}\right)\left[\mathcal{C} \in \mathbf{M i n}_{\psi^{\prime}} \mathbf{E x}\right]\right]$. We do not know at this point whether, $(\exists \mathcal{C})(\exists$ Gödel Numbering $\psi)\left[\mathcal{C} \in \operatorname{Min}_{\psi}\right.$ CoLearn $\wedge(\forall$ Kolmogorov Numbering $\left.\eta)\left[\mathcal{C} \notin \operatorname{Min}_{\eta} \mathbf{E x}\right]\right]$. However,

Theorem $28(\exists \mathcal{C})(\exists$ Gödel Numbering $\psi)\left[\mathcal{C} \in \operatorname{Min}_{\psi} \mathbf{E x} \wedge(\forall\right.$ Kolmogorov Numbering $\left.\eta)\left[\mathcal{C} \notin \operatorname{Min}_{\eta} \mathbf{E x}\right]\right]$.

Proof. Let $F$ be a partial recursive function, such that $F(k, i, x)$, denotes the output of the $i$-th program in the $k$-th numbering on input $x$. Note that $F(k, \cdot, \cdot)$ is a numbering, and $F(k, i, \cdot)$ denotes the function computed by the $i$-th program in this numbering.

We will construct a recursive function $g(k, i, j)$ using parameterized recursion theorem. We will have that, for all $k, i, j$, zeroext $\left(\varphi_{g(k, i, j)}\right) \in \operatorname{ZEROSTAR}$.

Intuitively our plan is as follows:

(A) We try to make zeroext $\left(\varphi_{g(k, i, j)}\right) \notin \operatorname{Min}_{F(k,, \cdot)} \mathbf{E x}\left(\mathbf{M}_{i}\right)$. For this we use a technique used by Chen [Che82] to show that ZEROSTAR $\notin$ MEx (we refer the reader to Chen [Che82] for definition of MEx-identification). However, since we do not know the reduction from $\varphi_{g(k, i, \cdot)}$ to the Kolmogorov numbering $\mathbf{F}(k, \cdot, \cdot)$, we may not always be successful. We will however be successful for large enough $j$ (where large enough depends only on $i$ and $k$ ).

(B) Using $g$, we will construct a class $\mathcal{C}$ (which contains, for each $i, k$, infinitely many of zeroext $\left.\left(\varphi_{g(k, i, \cdot)}\right)\right)$ and a Gödel numbering $\psi$ such that $\mathcal{C} \in \mathbf{M i n}_{\psi} \mathbf{E x}$. For this purpose we need to code $\langle k, i, j\rangle$ in $\varphi_{g(k, i, j)}$.

This would prove the theorem. We now proceed formally.

By parameterized s-m-n theorem [Rog67] there exists a recursive 1-1 function $g$ such that $\varphi_{g(k, i, j)}$, may be defined as follows. 
Begin definition of $\varphi_{g(k, i, j)}$

Let Cancel $=\emptyset$.

Let $\left.\varphi_{g(k, i, j}\right)(0)=\langle k, i, j\rangle$.

Let $x_{s}$ denote the least $x$ such that $\varphi_{g(k, i, j)}(x)$ has not been defined before stage $s$. Go to stage 0 .

Begin stage $s$

0. Dovetail steps 1 and 2, until one of them succeeds. If step 1 succeeds (before step 2 , if ever) then go to step 3. If step 2 succeeds (before step 1, if ever) then go to step 4 .

1. Search for an $l<j^{2}$, such that $l \notin$ Cancel and $F\left(k, l, x_{s}\right) \downarrow$.

2. Suppose $f=\operatorname{zeroext}\left(\varphi_{g(k, i, j)}\left[x_{s}\right]\right)$. Search for $m>x_{s}$, such that $\mathbf{M}_{i}(f[m]) \geq j^{2}$.

3. Let $l$ be as found in step 1 . Let $\varphi_{g(k, i, j)}\left(x_{s}\right)=F\left(k, l, x_{s}\right)+1$.

Let Cancel $=$ Cancel $\cup\{l\}$.

Go to stage $s+1$.

4. Let $m$ be as found in step 2. For $x_{s} \leq x \leq m$, let $\varphi_{g(k, i, j)}(x)=0$.

Go to stage $s+1$.

End stage $s$

End definition of $\varphi_{g(k, i, j)}$

Claim 29 Suppose $F(k, \cdot, \cdot)$ is a Kolmogorov numbering. Then for all $i$, for all but finitely many $j$, zeroext $\left(\varphi_{g(k, i, j)}\right) \notin \operatorname{Min}_{F(k, \cdot, \cdot)} \mathbf{E x}\left(\mathbf{M}_{i}\right)$.

Proof. Fix $\mathbf{M}_{i}$. Suppose $F(k, \cdot, \cdot)$ is a Kolmogorov numbering. Then for all but finitely many $j, \operatorname{MinProg}_{F(k,, \cdot)}\left(\varphi_{g(k, i, j)}\right)<j^{2}$.

Suppose $j$ is such that $\operatorname{MinProg}_{F(k, \cdot, \cdot)}\left(\varphi_{g(k, i, j)}\right)<j^{2}$. Let $f=\operatorname{zeroext}\left(\varphi_{g(k, i, j)}\right)$. We will show that $f \notin \operatorname{Min}_{F(k, \cdot, \cdot)} \mathbf{E x}\left(\mathbf{M}_{i}\right)$. Consider the following two cases.

Case 1: $(\stackrel{\infty}{\exists} n)\left[\mathbf{M}_{i}(f[n]) \geq j^{2}\right]$.

In this case, due to the success of step 2 in the construction of $\varphi_{g(k, i, j)}$ infinitely often, we have, $f=\varphi_{g(k, i, j)} \notin \mathbf{M i n}_{F(k, \cdot, \cdot)} \mathbf{E x}\left(\mathbf{M}_{i}\right)$.

Case 2: $(\stackrel{\forall}{\forall} n)\left[\mathbf{M}_{i}(f[n])<j^{2}\right]$.

By the construction of $\varphi_{g(k, i, j)}$, it follows that $\left(\forall l<j^{2}\right)[l \in$ Cancel $\vee$ $F(k, l, \cdot)$ is not total ]. Therefore, $\left(\forall l<j^{2}\right)[F(k, l, \cdot) \neq f]$. Thus $f \notin$ $\operatorname{Min}_{F(k, \cdot, \cdot)} \operatorname{Ex}\left(\mathbf{M}_{i}\right)$.

Define $\mathcal{C}_{\langle k, i, j\rangle}$ as follows.

$$
\mathcal{C}_{\langle k, i, j\rangle}=\left\{\operatorname{zeroext}\left(\varphi_{g(k, i, j)}[x]\right) \mid x=1 \vee \varphi_{g(k, i, j)}(x-1) \downarrow \neq 0\right\}
$$


Note that zeroext $\left(\varphi_{g(k, i, j)}\right) \in \mathcal{C}_{\langle k, i, j\rangle}$, and $\operatorname{card}\left(\mathcal{C}_{\langle k, i, j\rangle}\right) \leq j^{2}+1$ (since step 1 can succeed only $j^{2}$ many times). Moreover the functions in $\mathcal{C}_{\langle k, i, j\rangle}$ are 1-1 enumerable effectively in $\langle k, i, j\rangle$.

Let

$$
S=\left\{\langle k, i, j\rangle \mid(\forall p \leq \sqrt{j})\left[\varphi_{p}(0) \neq\langle k, i, j\rangle\right]\right\}
$$

Let

$$
\mathcal{C}=\bigcup_{\langle k, i, j\rangle \in S} \mathcal{C}_{\langle k, i, j\rangle}
$$

Note that for each $k, i$, there exist infinitely many $j$, such that $\mathcal{C}_{\langle k, i, j\rangle} \subseteq \mathcal{C}$. Thus, for all $i, k$, there exists infinitely many $j$ such that zeroext $\left(\varphi_{g(k, i, j)}\right) \in \mathcal{C}$. It follows from Claim 29 that, $\left(\forall\right.$ Kolmogorov Numbering $\left.\psi^{\prime}\right)\left[\mathcal{C} \notin \mathbf{M i n}_{\psi^{\prime}} \mathbf{E x}\right]$.

We now construct a Gödel numbering $\psi$, such that $\mathcal{C} \in \mathbf{M i n}_{\psi} \mathbf{E x}$. Let $\operatorname{gap}(l)=l^{4}+2$. Let $h(0)=0$; for $l \in N$, let $h(l+1)=1+h(l)+g a p(l)$.

Let $\psi_{h(l+1)}=\varphi_{l}$. Note that this makes $\psi$ a Gödel numbering.

For $h(l)<x<h(l+1), \psi_{x}$ is defined as follows. If $\varphi_{l}(0) \downarrow=\langle k, i, j\rangle$ and $\operatorname{card}\left(\mathcal{C}_{\langle k, i, j\rangle}\right) \geq$ $x-h(l)$, then let $\psi_{x}=(x-h(l))$-th function in $\mathcal{C}_{\langle k, i, j\rangle}$ (in some standard, 1-1, effective in $k, i, j$, enumeration of $\left.\mathcal{C}_{\langle k, i, j\rangle}\right)$. Otherwise let $\psi_{x}$ be the everywhere undefined function.

\section{Claim $30 \mathcal{C} \in \operatorname{Min}_{\psi} \mathbf{E x}$.}

Proof. Suppose, $\langle k, i, j\rangle \in S$. Suppose $l$ is such that $\varphi_{l}(0)=\langle k, i, j\rangle$. Then we have $l>\sqrt{j}$. Thus, $j^{2}+1 \leq l^{4}+1$. Hence, $\mathcal{C}_{\langle k, i, j\rangle} \subseteq\left\{\psi_{r} \mid h(l)<r<h(l+1)\right\}$.

Thus for all $f \in \mathcal{C}$, we have:

$$
h(l)<\operatorname{MinProg}_{\psi}(f)<h(l+1)
$$

where $l=\min \left(\left\{r \mid \varphi_{r}(0)=f(0)\right\}\right)$.

Thus, in particular, (A) for all $f \in \mathcal{C}, \operatorname{MinProg}_{\psi}(f)$ is not of the form $h(l)$, for any $l$. Moreover the construction of $\psi$ gives us the following: (B) if $h(l)<x<h(l+1)$, then either $\psi_{x}$ is total or $\psi_{x}$ is everywhere undefined.

Using properties (A) and (B) of $\psi$, it is easy to show that $\mathcal{C} \in \mathbf{M i n}_{\psi} \mathbf{E x}$.

Recall that in every Kolmogorov numbering $\psi, \mathbf{M i n}_{\psi}$ FIN, $\mathbf{M i n}_{\psi}$ CoLearn, $\mathbf{M i n}_{\psi} \mathbf{E x}$ are separated. However, as the following theorem shows, in Gödel numbering this may not be the case.

Theorem 31 For all $\alpha_{1}, \alpha_{2}, \alpha_{3} \in\{=, \subset\}$, it is possible to construct a Gödel Numbering $\eta$ such that FINITE $\alpha_{1} \operatorname{Min}_{\eta}$ FIN $\alpha_{2} \operatorname{Min}_{\eta}$ CoLearn $\alpha_{3} \operatorname{Min}_{\eta} \mathbf{E x}$.

ProOF. The idea is essentially to interleave the diagonalizations for the relevant proper subset construction with the Gödel numbering in which no infinite set of functions is MinEx-identifiable.

Lemmas 34, 35 and 38 below give us (non-universal) computable numberings $\psi^{1}, \psi^{2}, \psi^{3}$, and monotone increasing recursive functions $g^{1}, g^{2}, g^{3}$ such that properties (A) to (G) below are satisfied. 
Below let $\mathcal{C}^{1}=\left\{\psi_{j}^{1} \mid \psi_{j}^{1} \in \mathcal{R}\right\}, \mathcal{C}^{2}=\left\{\psi_{j}^{2} \mid \psi_{j}^{2} \in \mathcal{R}\right\}$, and $\mathcal{C}^{3}=\left\{\psi_{j}^{3} \mid \psi_{j}^{3} \in \mathcal{R}\right\}$.

(A) $\mathcal{C}^{1}, \mathcal{C}^{2}, \mathcal{C}^{3}$ are infinite and pairwise disjoint.

(B) $\mathcal{C}^{1} \in \operatorname{Min}_{\psi^{1}} \mathbf{F I N}$.

(C) $\mathcal{C}^{2} \in \operatorname{Min}_{\psi^{2}}$ CoLearn.

(D) $\mathcal{C}^{3} \in \operatorname{Min}_{\psi^{3}} \mathbf{E x}$.

(E) No infinite subset of $\mathcal{C}^{2}$ belongs to $\mathbf{M i n}_{\psi^{2}}$ FIN.

(F) No infinite subset of $\mathcal{C}^{3}$ belongs to $\mathbf{M i n}_{\psi^{3}}$ CoLearn.

(G) For each $i \in\{1,2,3\}$, there exist infinitely many $j \in N$ such that, $\operatorname{card}\left(\left\{\psi_{r}^{i} \mid r \leq\right.\right.$ $\left.\left.g^{i}(j) \wedge \psi_{r}^{i} \in \mathcal{C}^{i}\right\}\right)>2 j$.

Using the above numberings, we construct a Gödel numbering $\eta$ satisfying the theorem.

Suppose $\beta$ is the Gödel numbering in which no infinite class of functions can be $\mathbf{M i n}_{\beta} \mathbf{E x}$-identified. Intuitively we would like to interleave the numberings $\beta, \psi^{1}, \psi^{2}, \psi^{3}$, so that, for $i \in\{1,2,3\}, g^{i}(j)$-th program in $\psi^{i}$ appears before $j$-th program in $\beta$ in the interleaving.

For this purpose let $H$ be a $1-1$ function from $\{(x, y) \mid 1 \leq x \leq 4 \wedge y \in N\}$ to $N$, such that the following two properties are satisfied.

(1) For $i \in\{1,2,3\}, H\left(i, g^{i}(j)\right)<H(4, j)$.

(2) For each $i \in\{1,2,3,4\}, H(i, j)$ is a monotone increasing function of $j$.

Note that such a function $H$ can be easily constructed. For $i \in\{1,2,3,4\}$ and $k \in N$, let $\eta_{H(i, k)}$ be defined as follows.

$$
\eta_{H(i, k)}(x)= \begin{cases}\beta_{k}(x), & \text { if } i=4 ; \\ \psi_{k}^{i}(x), & \text { if } i \in\{1,2,3\} \text { and } \alpha_{i} \text { is } \subset ; \\ \uparrow, & \text { if } i \in\{1,2,3\} \text { and } \alpha_{i} \text { is }=.\end{cases}
$$

Claim 32 (a) If $\mathcal{C} \subseteq \mathbf{M i n}_{\eta} \mathbf{F I N}(\mathbf{M})$, then for all but finitely many $f \in \mathcal{C}, \mathbf{M}(f) \in$ $\{H(i, k) \mid k \in N \wedge i=1\}$.

(b) If $\mathcal{C} \subseteq \mathbf{M i n}_{\eta} \operatorname{CoLearn}(\mathbf{M})$, then for all but finitely many $f \in \mathcal{C}, \mathbf{M}(f)$ coconverges to a member of $\{H(i, k) \mid k \in N \wedge i \in\{1,2\}\}$.

(c) If $\mathcal{C} \subseteq \mathbf{M i n}_{\eta} \mathbf{E x}(\mathbf{M})$, then for all but finitely many $f \in \mathcal{C}, \mathbf{M}(f) \in\{H(i, k) \mid k \in$ $N \wedge i \in\{1,2,3\}\}$.

Proof. Note that if, $H(i, k)=\operatorname{MinProg}_{\eta}(f)$, then $k=\operatorname{MinProg}_{\psi^{i}}(f)$, where we let $\psi^{4}=\beta$. Thus, the claim follows from the construction of $\eta$ and the facts that

(i) no infinite subset of $\mathcal{R}$ belongs to $\mathbf{M i n}_{\beta} \mathbf{E x}$,

(ii) no infinite subset of $\mathcal{C}^{3}$ belongs to $\mathbf{M i n}_{\psi^{3}}$ CoLearn, and

(iii) no infinite subset of $\mathcal{C}^{2}$ belongs to $\mathbf{M i n}_{\psi^{2}}$ FIN. 
Claim 33 The following hold.

(a) Suppose $\alpha_{1}$ is $\subset$. Then $\left\{\psi_{i}^{1} \mid \psi_{i}^{1} \in \mathcal{R} \wedge \operatorname{MinProg}_{\eta}\left(\psi_{i}^{1}\right)=H(1, i)\right\} \in \mathbf{M i n}_{\eta} \mathbf{F I N}$. Moreover, $\left\{\psi_{i}^{1} \mid \psi_{i}^{1} \in \mathcal{R} \wedge \operatorname{MinProg}_{\eta}\left(\psi_{i}^{1}\right)=H(1, i)\right\}$ is infinite.

(b) Suppose $\alpha_{2}$ is $\subset$. Then $\left\{\psi_{i}^{2} \mid \psi_{i}^{2} \in \mathcal{R} \wedge \operatorname{MinProg}_{\eta}\left(\psi_{i}^{2}\right)=H(2, i)\right\} \in \operatorname{Min}_{\eta}$ CoLearn. Moreover, $\left\{\psi_{i}^{2} \mid \psi_{i}^{2} \in \mathcal{R} \wedge \operatorname{MinProg}_{\eta}\left(\psi_{i}^{2}\right)=H(2, i)\right\}$ is infinite.

(c) Suppose $\alpha_{3}$ is $\subset$. Then $\left\{\psi_{i}^{3} \mid \psi_{i}^{3} \in \mathcal{R} \wedge \operatorname{MinProg}_{\eta}\left(\psi_{i}^{3}\right)=H(3, i)\right\} \in \mathbf{M i n}_{\eta} \mathbf{E x}$. Moreover, $\left\{\psi_{i}^{3} \mid \psi_{i}^{3} \in \mathcal{R} \wedge \operatorname{MinProg}_{\eta}\left(\psi_{i}^{3}\right)=H(3, i)\right\}$ is infinite.

Proof. We show part (a). Proof of other parts are similar. Suppose $\alpha_{1}$ is $\subset$. Suppose M is such that $\mathcal{C}^{1} \subseteq \mathbf{M i n}_{\psi^{1}} \mathbf{F I N}(\mathbf{M})$. Let $\mathbf{M}^{\prime}$ be defined as follows. $\mathbf{M}^{\prime}(\sigma)=H(1, \mathbf{M}(\sigma))$. Clearly, $\left\{\psi_{i}^{1} \mid \psi_{i}^{1} \in \mathcal{R} \wedge \operatorname{MinProg}_{\eta}\left(\psi_{i}^{1}\right)=H(1, i)\right\} \in \mathbf{M i n}_{\psi^{1}} \mathbf{F I N}\left(\mathbf{M}^{\prime}\right)$.

Now, since $\mathcal{C}^{1}, \mathcal{C}^{2}, \mathcal{C}^{3}$ are pairwise disjoint, we have, for infinitely many $j$, $\operatorname{card}(\{i \mid$ $\left.\left.i \leq g^{1}(j) \wedge \psi_{i}^{1} \in \mathcal{C}^{1} \wedge H(1, i)=\operatorname{MinProg}_{\eta}\left(\psi_{i}^{1}\right)\right\}\right) \geq 2 j+1-j$.

It follows that $\left\{\psi_{i}^{1} \mid \psi_{i}^{1} \in \mathcal{R} \wedge \operatorname{MinProg}_{\eta}\left(\psi_{i}^{1}\right)=H(1, i)\right\}$ is infinite.

Theorem follows from the above two claims.

Lemma 34 There exists (non-universal) computable numbering $\psi^{1}$ and monotone increasing recursive function $g^{1}$, which satisfy properties $(A)$ to $(C)$ below.

Below let $\mathcal{C}^{1}=\left\{\psi_{j}^{1} \mid \psi_{j}^{1} \in \mathcal{R}\right\}$.

(A) For all $f \in \mathcal{C}^{1}, f(0)=1$.

(B) $\mathcal{C}^{1} \in \operatorname{Min}_{\psi^{1}} \mathbf{F I N}$.

(C) There exists infinitely many $j \in N$ such that, $\operatorname{card}\left(\left\{\psi_{r}^{1} \mid r \leq g^{1}(j) \wedge \psi_{r}^{1} \in \mathcal{C}^{1}\right\}\right)>$ $2 j$.

Proof. Let

$$
\psi_{k}^{1}(x)= \begin{cases}1, & \text { if } x=0 \\ k, & \text { otherwise }\end{cases}
$$

Let $g^{i}(j)=2 j+2$. It is easy to verify that the properties (A) to (C) are satisfied.

Lemma 35 There exists (non-universal) computable numbering $\psi^{2}$ and monotone increasing recursive function $g^{2}$, which satisfy properties (A) to (D) below.

Below let $\mathcal{C}^{2}=\left\{\psi_{j}^{2} \mid \psi_{j}^{2} \in \mathcal{R}\right\}$.

(A) For all $f \in \mathcal{C}^{2}, f(0)=2$.

(B) $\mathcal{C}^{2} \in \operatorname{Min}_{\psi^{2}}$ CoLearn.

(C) No infinite subset of $\mathcal{C}^{2}$ belongs to $\mathbf{M i n}_{\psi^{2}}$ FIN.

(D) There exists infinitely many $j \in N$ such that, $\operatorname{card}\left(\left\{\psi_{r}^{2} \mid r \leq g^{2}(j) \wedge \psi_{r}^{2} \in \mathcal{C}^{2}\right\}\right)>$ $2 j$. 
ProOF. Let $\sigma_{0}, \sigma_{1}, \ldots$ be an 1-1 enumeration of all elements of INIT. We assume without loss of generality that $\left|\sigma_{i}\right| \leq i$. Let $X$ be a recursive function from $N^{2}$ to $N$ such that the following properties are satisfied (note that such an $X$ can easily be constructed).

(1) $(\forall j)\left[\sigma_{X(j, 0)}=\{(0,2),(1, j)\}\right]$.

(2) $(\forall j, l)\left[\sigma_{X(j, l)} \subseteq \sigma_{X(j, l+1)}\right]$.

(3) $(\forall j)\left[\lim _{l \rightarrow \infty} X(j, l) \downarrow\right]$.

(4) $(\forall j, l)\left[X(j, l) \neq X(j, l+1) \Rightarrow\left|\sigma_{X(j, l+1)}\right|<l\right]$.

(5) For any given $j$, suppose $\tau_{j}=\sigma_{\lim _{l \rightarrow \infty} X(j, l)}$. Then $(\forall k<j)\left[\left(\exists \tau^{\prime} \supseteq \tau_{j}\right)\left[\mathbf{M}_{k}\left(\tau^{\prime}\right) \neq\right.\right.$ $\left.?] \Rightarrow\left[\mathbf{M}_{k}\left(\tau_{j}\right) \neq ?\right]\right]$.

Intuitively, $\tau_{j}$ above denotes a sequence such that all $\mathbf{M}_{k}, k<j$, which output program on some extension of $\tau_{j}$, output a program on $\tau_{j}$. Conditions, (1) - (4) above just impose some restrictions on the search of such $\tau_{j}$, which is used for implementing the diagonalization.

For $j \in N$, let $l_{j}$ be the least value of $l$ such that $\left(\forall l^{\prime}>l\right)\left[X(j, l)=X\left(j, l^{\prime}\right)\right]$ (note that since $\lim _{t \rightarrow \infty} X(j, t) \downarrow, l_{j}$ is well defined). Intuitively, $l_{j}$ is just the convergence point for $X(j, \cdot)$.

Define $h$ as follows:

$h(0)=0 . h(k+1)=h(k)+3 k+2$.

For $r \in N, r<3 *\langle j, l\rangle+2$, let

$$
f_{h(\langle j, l\rangle)+r}(x)= \begin{cases}\sigma_{X(j, l)}(x), & \text { if } x<\left|\sigma_{X(j, l)}\right| \\ h(\langle j, l\rangle)+r+1, & \text { if } x=\left|\sigma_{X(j, l)}\right| \\ 0, & \text { otherwise }\end{cases}
$$

Now, for $r<3 *\langle j, l\rangle+2$, let $\psi_{h(\langle j, l\rangle)+r}^{2}$ be defined so that the following two properties are satisfied:

(a) $\psi_{h(\langle j, l\rangle)+r}^{2} \subseteq f_{h(\langle j, l\rangle)+r}$.

(b) $\psi_{h(\langle j, l\rangle)+r}^{2}=f_{h(\langle j, l\rangle)+r}$ iff $\left[l=l_{j} \wedge(\forall m<j)\left[\mathbf{M}_{m}\right.\right.$ on $f_{h(\langle j, l\rangle)+r}$ does not finitely converge to $h(\langle j, l\rangle)+r]]$.

Intuitively, aim of part (b) is to make $\psi_{h(\langle j, l\rangle)+r}^{2}$ total iff the convergence point of $X(j, \cdot)$ is $l$, and no machine $\mathbf{M}_{m}, m<j$, finitely converges on $f_{h(\langle j, l\rangle)+r}$ to $h(\langle j, l\rangle)+r$.

Let $g^{2}(k)=h(k+1)$. We now show that $\psi^{2}$ and $g^{2}$ satisfy the conditions for the lemma.

Claim 36 No machine $\mathbf{M i n}_{\psi^{2}} \mathbf{F I N}$-identifies an infinite subset of $\mathcal{C}^{2}=\left\{\psi_{i}^{2} \mid \psi_{i}^{2} \in \mathcal{R}\right\}$.

Proof. Clearly, by definition of $\psi_{h(\langle j, l\rangle)+r}^{2}$, where $r<3 *\langle j, l\rangle+2$, we have

(1) $\psi_{h(\langle j, l\rangle)+r}^{2} \in \mathcal{R} \Rightarrow l=l_{j}$.

(2) For all $j$, for all $m<j, \mathbf{M}_{m}$ does not $\mathbf{M i n}_{\psi^{2}} \mathbf{F i n}$-identify any function in $\left\{\psi_{h(\langle j, l\rangle)+r}^{2} \mid r<3 *\langle j, l\rangle+2 \wedge \psi_{h(\langle j, l\rangle)+r}^{2} \in \mathcal{R}\right\}$.

The claim follows from above.

Claim 37 Let $\mathcal{C}^{2}=\left\{\psi_{i}^{2} \mid \psi_{i}^{2} \in \mathcal{R}\right\}$. Then, for infinitely many $k \in N$, $\operatorname{card}\left(\left\{\psi_{r}^{2} \mid r \leq\right.\right.$ $\left.\left.g^{2}(k) \wedge \psi_{r}^{2} \in \mathcal{C}^{2}\right\}\right)>2 k$. Moreover, $\mathcal{C}^{2} \in \operatorname{Min}_{\psi^{2}}$ CoLearn. 
Proof. For all $j$, for $r<3 *\left\langle j, l_{j}\right\rangle+2, \sigma_{X\left(j, l_{j}\right)} \subseteq f_{h\left(\left\langle j, l_{j}\right\rangle\right)+r}$. Moreover, by property (5) of $X$, for all $m<j$, if $\mathbf{M}_{m}$ outputs a program on $f_{h(\langle j, l\rangle)+r}$, then $\mathbf{M}_{m}$ outputs a program on $\sigma_{X\left(j, l_{j}\right)}$. It thus follows from the construction of $\psi^{2}$ that, there exist at least $3 *\left\langle j, l_{j}\right\rangle+2-j$ distinct values for $r<3 *\left\langle j, l_{j}\right\rangle+2$, such that $\psi_{h\left(\left\langle j, l_{j}\right\rangle\right)+r}^{2}=f_{h\left(\left\langle j, l_{j}\right\rangle\right)+r} \in \mathcal{R}$. Now, since $f_{i}$ 's are distinct, it follows that, for all $j$,

$$
\operatorname{card}\left(\left\{\psi_{r}^{2} \mid r \leq g^{2}\left(\left\langle j, l_{j}\right\rangle\right) \wedge \psi_{r}^{2} \in \mathcal{C}^{2}\right\}\right) \geq 3 *\left\langle j, l_{j}\right\rangle+2-j>2 *\left\langle j, l_{j}\right\rangle
$$

(recall that according to the assumption on our pairing function, $\langle i, j\rangle \geq \max (\{i, j\})$ ).

We now give a machine $\mathbf{M}$ such that $\mathcal{C}^{2} \subseteq \operatorname{Min}_{\psi^{2}} \operatorname{CoLearn}(\mathbf{M})$. For this it is sufficient to construct a machine $\mathbf{M}$ such that $\mathbf{M}$ on $f_{k}$ co-converges to $k$.

First note that $l>\left|\sigma_{X(j, l)}\right|-3$ (we need -3 just to address the case of $l=0$ ). This implies (from the definition of $f_{h(\langle j, l\rangle)+r}$ ) that, for all $j$, for all $r<3 *\langle j, l\rangle+2$,

$$
h(\langle j, l\rangle)+r \geq\langle j, l\rangle \geq l \geq\left|\sigma_{X(j, l)}\right|-3 \geq \max \left(\left\{x \mid f_{h(\langle j, l\rangle)+r}(x) \neq 0\right\}\right)-4
$$

Also note that, for all $k, f_{k}\left(\max \left(\left\{x \mid f_{k}(x) \neq 0\right\}\right)\right)=k+1$. Let $\mathbf{M}$ be such that:

$$
\mathbf{M}(\sigma)= \begin{cases}?, & \text { if }|\sigma| \leq 5 ; \\ \min (N-\{\sigma(\max (\{x \mid \sigma(x) \neq 0\}))-1\}), & \text { otherwise. }\end{cases}
$$

From the discussion above, it is easy to see that $\mathbf{M}$ on $f_{k}$ co-converges to $k$. Thus $\mathcal{C}^{2} \subseteq \operatorname{Min}_{\psi^{2}} \operatorname{CoLearn}(\mathrm{M})$.

Lemma follows from the above two claims.

Lemma 38 There exists (non-universal) computable numbering $\psi^{3}$ and monotone increasing recursive function $g^{3}$, which satisfy properties $(A)$ to $(D)$ below.

Below let $\mathcal{C}^{3}=\left\{\psi_{j}^{3} \mid \psi_{j}^{3} \in \mathcal{R}\right\}$.

(A) For all $f \in \mathcal{C}^{3}, f(0)=3$.

(B) $\mathcal{C}^{3} \in \operatorname{Min}_{\psi^{3}} \mathbf{E x}$.

(C) No infinite subset of $\mathcal{C}^{3}$ belongs to $\mathbf{M i n}_{\psi^{3}}$ CoLearn.

(D) There exists infinitely many $j \in N$ such that, $\operatorname{card}\left(\left\{\psi_{r}^{3} \mid r \leq g^{3}(j) \wedge \psi_{r}^{3} \in \mathcal{C}^{3}\right\}\right)>$ $2 j$.

ProOF. Let

$$
f_{i}(x)= \begin{cases}3, & \text { if } x=0 \\ i, & \text { if } x=1 \\ 0, & \text { otherwise }\end{cases}
$$

Let $h(0)=0 ; h(i+1)=h(i)+i+1$.

For $h(i) \leq j<h(i+1)$, let $\psi_{j}^{3}$ be defined in such a way that the following two properties are satisfied.

(a) $\psi_{j}^{3} \subseteq f_{i}$.

(b) $\psi_{j}^{3}$ is total iff $(\forall m<i)\left[F_{\mathbf{M}_{m}}\left(\Lambda, f_{i}\right) \cap\{y \mid y<h(i+1)\} \neq\{y \mid y<h(i+1) \wedge y \neq j\}\right]$. 
Note that one can easily construct such $\psi^{3}$. Let $g^{3}(k)=h(2 k+1)$.

From the properties of $\psi^{3}$ discussed above, it is easy to verify that, for all $i$, there exists a $j, h(i) \leq j<h(i+1)$, such that $\psi_{j}^{3}=f_{i}$. Moreover, for all $m<i, f_{i} \notin$ $\operatorname{Min}_{\psi^{3}} \operatorname{CoLearn}\left(\mathbf{M}_{m}\right)$. Thus properties (A), (C) and (D) of lemma are satisfied.

Note that in the limit it is easy to verify, if $(\forall m<i)\left[F_{\mathbf{M}_{m}}\left(\Lambda, f_{i}\right) \cap\{y \mid y<h(i+1)\} \neq\right.$ $\{y \mid y<h(i+1) \wedge y \neq j\}]$. Thus in the limit, for each $f_{i}$, one can find the minimum $j$, such that $h(i) \leq j<h(i+1)$, and $(\forall m<i)\left[F_{\mathbf{M}_{m}}\left(\Lambda, f_{i}^{3}\right) \cap\{y \mid y<h(i+1)\} \neq\{y \mid y<\right.$ $h(i+1) \wedge y \neq j\}]$. It follows that $\mathcal{C}^{3} \in \mathbf{M i n}_{\psi} \mathbf{E x}$.

\section{Conclusions}

In this paper we studied identification by minimal grammars for FIN, CoLearn, and Ex-identification criteria in Kolmogorov and Gödel numberings. We showed that for every Kolmogorov Numbering, $\psi$, FINITE, $\mathbf{M i n}_{\psi}$ FIN, $\mathbf{M i n}_{\psi}$ CoLearn, and $\mathbf{M i n}_{\psi} \mathbf{E x}$ are distinct. We also showed that every possible relationship consistent with FINITE $\subseteq \mathbf{M i n}_{\psi} \mathbf{F I N} \subseteq \mathbf{M i n}_{\psi}$ CoLearn $\subseteq \mathbf{M i n}_{\psi}$ Ex can be realized for some Gödel numbering $\psi$. In addition we compared minimal identification in Kolmogorov numberings vis-a-vis Gödel numberings.

\section{Acknowledgements}

First Author was supported in part by Latvian Council of Science Grant 93.599 and NSF Grant 9119540. This work was done in part while the first author was visiting the Institute of Systems Science, at the National University of Singapore. A preliminary version of this paper appeared in Computational Learning Theory, Second European Conference, EuroColt' 95 [FJ95].

\section{References}

[BB75] L. Blum and M. Blum. Toward a mathematical theory of inductive inference. Information and Control, 28:125-155, 1975.

[Blu67] M. Blum. A machine independent theory of the complexity of recursive functions. Journal of the ACM, 14:322-336, 1967.

[Cas74] J. Case. Periodicity in generations of automata. Mathematical Systems Theory, 8:15-32, 1974.

[Che81] K. Chen. Tradeoffs in Machine Inductive Inference. PhD thesis, SUNY at Buffalo, 1981.

[Che82] K. Chen. Tradeoffs in inductive inference of nearly minimal sized programs. Information and Control, 52:68-86, 1982. 
[CS83] J. Case and C. Smith. Comparison of identification criteria for machine inductive inference. Theoretical Computer Science, 25:193-220, 1983.

[FJ95] R. Freivalds and S. Jain. Kolmogorov numberings and minimal identification. In Paul Vitanyi, editor, Computational Learning Theory, Second European Conference, EuroCOLT'95, Barcelona, Spain, pages 182-195. Springer-Verlag, March 1995. Lecture Notes in Artificial Intelligence 904.

[FKS94] R. Freivalds, M. Karpinski, and C. H. Smith. Co-learning of total recursive functions. In Proceedings of the Seventh Annual Conference on Computational Learning Theory, New Brunswick, New Jersey, pages 190-197. ACM Press, July 1994.

[Fre75] R. Freivalds. Minimal Gödel numbers and their identification in the limit. In Proceedings of the International Conference on Mathematical Foundations of Computer Science, Marianske Lazne, pages 219-225. Springer-Verlag, 1975. Lecture Notes in Computer Science 32.

[Fre91] R. Freivalds. Inductive inference of recursive functions: Qualitative theory. In J. Barzdins and D. Bjorner, editors, Baltic Computer Science. Lecture Notes in Computer Science 502, pages 77-110. Springer-Verlag, 1991.

[Gol67] E. M. Gold. Language identification in the limit. Information and Control, 10:447474, 1967.

[HU79] J. Hopcroft and J. Ullman. Introduction to Automata Theory, Languages, and Computation. Addison-Wesley Publishing Company, 1979.

[OSW86] D. Osherson, M. Stob, and S. Weinstein. Systems that Learn, An Introduction to Learning Theory for Cognitive and Computer Scientists. MIT Press, Cambridge, Mass., 1986.

[Rog67] H. Rogers. Theory of Recursive Functions and Effective Computability. McGraw-Hill, New York, 1967. Reprinted by MIT Press, Cambridge, Massachusetts in 1987. 